\title{
Expression of pBac 3xP3 EGFP and pBac A3 EGFP Vector in Transgenic Spodoptera litura Fabricius
}

\author{
Zhongling Gong,Wenjie Xu \\ Gas group co., LTD of Shenzhen City ’ 518049 • china
}

\begin{abstract}
In this study, the actin A3 promoter was inserted into piggyBac and constructed into pBacA3, which was inserted into piggyBac. The promoter was composed of the promoter and nerve-specific 3xP3 promoter, the enhanced green fluorescent protein (EGFP) gene and the SV40 polyadenylation recognition sequence. EGFP and pBac3xP3 EGFP transposon were injected into the early fertilized eggs of Spodoptera litura to detect whether they were expressed. The results showed that both vectors could be expressed in moth eggs, and positive individuals were obtained. The results showed that the expression rate of pBacA3 EGFP was higher than that of pBac3xP3 EGFP, and the proportion of EGFP in hatching larvae was higher than that in the latter. The results showed that pBacA3 EGFP was more suitable for Spodoptera litura Transposable carrier. In vitro transient expression of the transgenic vector of Spodoptera litura was not only the first step necessary to successfully carry out the transgenic gene of Spodoptera litura, but also itself can be applied to the study of gene function, which laid the foundation for the genome research of Spodoptera litura.
\end{abstract}

Key words: Spodoptera litura, microinjection, pBacA3EGFP, pBac3xP3 EGFP, fluorescent expression.

\section{Introduction}

1.1 This topic is now at home and abroad research overview

Transgenic technology is a kind of important research method in the field of life sciences, which is the technology of introducing foreign genes into the biological genome and making it stable and able to be inherited to future generations. It is an important research field in the field of genetics and development, gene expression and regulation Have a broad application prospects. Insect transgenic technology can not only study gene function by overexpression or loss of function [12], but also the key to the development of insect bioreactor technology system [3].

So far, transposons found in insects have P, Tc1, Pogo and FP, ermes, mi-nos, hobo, mariner, piggyBac and so on. Among them, piggyBac transposon can be accurately cut and transcribed in organism chromosome, and has a wide range of applications, so piggyBac transposon carrier is widely used in Diptera, Lepidoptera, Coleoptera and membrane The most common carrier of insect transgenic research, it has been successfully applied to the transgenic research of Drosophila melanogaster, Drosophila melanogaster and silkworm [4]. Researchers in Japan have compiled a large number of recombinant proteins [5] by linking the human type III procollagen gene to the piggyBac transposon.

There are few reports on insect transgenic research using piggyBac transposons in China, and only some progress has been made in transgenic research of silkworm, while transgenic research of other insects is lagging behind. The study of transgenic gene of Spodoptera litura has not been reported.

1.2 Purpose and significance of this experiment

Spodoptera litura is a worldwide distribution of agricultural pests, in all continents have serious occurrence and damage records, resulting in vegetables, cotton, tobacco, sweet potato, corn and other crops and grass, turfgrass, green manure crops and other serious losses Agricultural production caused huge economic losses or social shocks (Qin Houguo, 2007) [6]. In view of the growing harassment of Spodoptera litura, the research and control of the insects have aroused great attention from domestic and foreign agricultural entomologists and plant protection workers and the extensive attention of the relevant government departments.

In this experiment, the gene was transferred by the method of microinjection with Spodoptera litura as the material, and the transposon piggyBac from Lepidoptera was used to use two different promoters - actin A3 promoter and ocular and nerve Specific 3xP3 promoter, the efficiency of different promoters was compared, and the optimal mode was selected to try to establish a highly efficient and stable transgenic Spodoptera litura technology system. In order to study the gene function of Spodoptera litura gene by transgenic technology, Mechanism and other basic research, and the use of transgenic technology to control the reproduction and harm of Spodoptera litura to provide a certain theoretical basis and practice.

\section{Materials and methods}

\subsection{Materials}

2.1.1 Experimental materials

The experimental insects are artificial breeding of Spodoptera littoralis under laboratory conditions, and the germplasm comes from the Institute of Insects of Sun Yat-sen University. Two kinds of piggybac (pA3EGFP and 3xP3EGFP) and helper from the South China Normal University College of Life Sciences 319 insect molecular biology laboratory.

2.1.2 Major reagents

Tris saturated phenol, formaldehyde, isopropanol, chloroform, plasmid extraction kit (QIAGEN, Shanghai Health), PCR amplification kit (Shanghai Health), Supercoli Marker (TAKARA)

2.1.3 Preparation of the main solution

Injection Microscope: $5 \mathrm{mM} \mathrm{KOH}, 0.5 \mathrm{mM} \mathrm{NaH2PO} 4$ was adjusted to $\mathrm{pH} 7.2$ with $0.1 \mathrm{M} \mathrm{NaOH}$ and sterilized by filtration.

$5 \mathrm{x}$ Tris-borate buffer (TBE): $54 \mathrm{~g}$ Tris base, $27.5 \mathrm{~g}$ boric acid, $20 \mathrm{ml} 0.5 \mathrm{~mol} / \mathrm{L}$ EDTA (pH 8.0)

\subsubsection{Major instruments}

The main equipment used in this experiment are: fluorescent inversion microscope (Nikon) and micro operation instrument, isoelectric focusing electrophoresis tank (USA), pull needle instrument (Germany), forging instrument (MF-79), grinding instrument (EG (GE Healthecare, USA), high-speed centrifuge (Flying Pigeon, China), 
ultra-low temperature refrigerator Thermo (Thermo Electron), etc., such as alcohol spray lamp, stereoscopic dissection mirror, electrophoresis, ordinary optical microscope, gel imaging system ImageQuant 300 The

2.2 Artificial rearing of Spodoptera litura

Egg mass disinfection: collection of eggs, into the concentration of $5 \%$ formaldehyde solution disinfection $5 \mathrm{~min}$, rinse with sterile water, dry naturally, into the incubator, to be hatched.

Larvae feeding: fresh culture medium for newly hatched larvae, until the larvae grow to three age, sub-box feeding. When the larvae are to the pupal stage, transfer to another plastic box containing about $10 \%$ of the sandy soil.

Adult (feathering - spawning): When the pupa's body color from reddish brown to dark brown, the pupa removed from the sand, into the wall with disinfectant paper transparent plastic box in the feather; after emergence, the male and female Moths are placed in proportion to the new plastic box, daily replacement of paper, with $10 \%$ of the honey. Observe the time of female oviposition, according to the experimental requirements, remove the time of the egg for microinjection.

Feeding conditions: temperature $25 \pm 1{ }^{\circ} \mathrm{C}$, relative humidity $70 \%-80 \%$, photoperiod $14 \mathrm{~h}: 10 \mathrm{~h}$ (light: dark).

2.3 Microinjection of genes

2.3.1 Collection of eggs of Spodoptera litura

Take the eclosion of the Spodoptera litura, according to the ratio of male and female 1: 1 into the transparent plastic box, the box drops $10 \%$ honey water and placed disinfection of rough paper to prepare the spawning. Mating and spawning were carried out at night, during the 21: 00-1: 00 period, every half hour observation, record the date of spawning and accurate spawning time. Take a few larger and spawning time known to the egg, to identify the health of the egg, assuming that the same egg of the egg development is synchronized, and the same egg as an independent treatment.

\subsubsection{Arrangement and fixation of eggs}

Select the female moths after a certain period of time within the health of the egg, with a soft brush gently brush off the surface of the egg scales, asked to try to brush clean. Slide with alcohol soak, dry naturally, in the glass on the fixed part of the egg coated with diluted 10 times after the paste, before its dry, with a soft brush to move the egg to the paste, and gently Its break up. In the vertical dissecting mirror, the anatomical needle to separate the eggs, and arranged neatly.

Figure 2-2 shows the fixed eggs and eggs before and after the extreme diagram

A: the eggs arranged on the slide; B: egg side of the photo (the top of the front pole, the lower end of the posterior pole); $\mathrm{C}$ eggs overlooking the photo (Figure for the front,

\subsubsection{Microinjection}

The plasmid was centrifuged at $10,000 \mathrm{r} / \min$ for $5 \mathrm{~min}$ at $4{ }^{\circ} \mathrm{C}$ on a freeze centrifuge and the mixed plasmid solution was then loaded directly onto a drawn injection tube with a diameter of less than $3 \mu \mathrm{m}$. After the eggs are fixed on the slide, the slides are placed on the stage,

adjust the focal length, and adjust the focal plane to the diameter of about 2 eggs above the slide at high magnification. Place the needle on the focus. Apply a certain pressure, swing the needle, see the formation of the tip near the surge and check the pinhole is blocked. Carefully adjust the micrometer to control the flow rate of the DNA solution to the right level. Insert the needle into the cytoplasm, slowly inject the DNA into the egg until the insertion port has a little liquid spill, and quickly pull out the tip to complete the injection.

2.3.4 Treatment after microinjection

A glass slide as far as possible within 40min to complete the injection of eggs placed in the outside should not exceed $1 \mathrm{~h}$. The slides were placed in $37 \%$ formaldehyde steam for $5 \mathrm{~min}$ disinfection, and then placed in a fully moist, sterile environment for $12 \mathrm{~h}$, until the wound was completely healed, transferred to the temperature of $25 \pm$ $1{ }^{\circ} \mathrm{C}$, relative humidity of $65 \% \sim 75 \%$ incubation in the environment for 3 days.

\subsubsection{Fluorescence screening}

After the injection, the eggs were observed every $24 \mathrm{~h}$ under the fluorescence microscope. Under the excitation of the blue light, the EGFP was observed and the GMO positive G0 individuals were screened. Each batch was injected into the unit.

2.4 Commonly used molecular technology

2.4.1 Extraction of plasmid DNA and genomic DNA

The experimental DNA was extracted with TIANGEN plasmid kit. The genomic DNA of the positive individuals was extracted with TIANGEN blood / cell / tissue genomic DNA extraction kit. For details, refer to the kit instructions.

\subsubsection{PCR amplification of the target gene}

The specific primers were designed according to the EGFP base sequence using Prmer 5 and sent to Shanghai Biotechnology Biotechnology Co., Ltd.

The forward primer P1 is: 5'-GTTCTCGTTGGGGTCTTTGCT$3^{\prime}$

The reverse primer P2 is: 5'-TCACAAGTTCAGCGTGTCCG-3

The experimental reaction with $20 \mu \mathrm{L}$ PCR reaction system was: 2X PCR mix $10 \mu \mathrm{L}$

Template DNA $1 \sim 5 \mu \mathrm{L}$

Primer $10.6 \mu \mathrm{L}$

Primers $20.6 \mu \mathrm{L}$

$\mathrm{DdH} 2 \mathrm{O} 8.8 \mu \mathrm{L}$

The conditions of the PCR reaction are determined according to the specific situation. Generally under the following conditions:

$94{ }^{\circ} \mathrm{C}$ pre-denaturation $3 \mathrm{~min}, 94^{\circ} \mathrm{C}$ denaturation $45 \mathrm{sec}, 52 \circ$ $\mathrm{C}$ refolding $45 \mathrm{sec}, 72^{\circ} \mathrm{C}$ extension $60 \mathrm{sec}$,

35 cycles at $72^{\circ} \mathrm{C}$ for $10 \mathrm{~min}$

2.4.3 Agarose gel electrophoresis

According to the size of the pre-isolated DNA fragment, the appropriate concentration of agarose gel was prepared with $0.5 \times \mathrm{TBE}$, and the gel was prepared on the gel plate of the micro electrophoresis bath.

After solidification, the gel is placed in an electrophoresis tank. Take appropriate sample and mix with $6 \times$ loading buffer and sample.

Electrophoresis buffer for the $0.5 \times \mathrm{TBE}$, the voltage of $50 \sim$ $100 \mathrm{~V}$, electrophoresis after $300 \mathrm{~nm} \mathrm{UV}$ transmission instrument detection.

\section{Results and analysis}


3.1 Expression of pBac3xP3 EGFP in Egg of Spodoptera litura Fabricius

With the same concentration of $\mathrm{pBac}$ and helper by $1: 1$ ratio of mixed, after microinjection, observed once every 24 hours, 48 hours after injection, the normal development of eggs, observed with fluorescence microscope, 100 times the microscope can be observed in part Hair green fluorescent eggs (Figure 3-1A). It can be seen from the figure, the embryos of Spodoptera litura in the blue fluorescence itself can send a weak yellow; and injected pBac3xP3 EGFP, the embryo can be made in a very strong green light, and fluorescence in the whole egg The distribution is not uniform (Figure 3-1B). Even at this time we can see that the embryo has begun to have the shape of the parasite, showing ' $U$ ' type of green fluorescence (Figure 3-1C-E).

In the 'U' type worm, there are two obvious green fluorescent expression, the initial judgment of the eye and the middle part of the larvae, may be Midgut, the specific expression of the need for further experimental verification later.

Figure 3-1 Expression of EGFP in eggs of Partial Spodoptera litura after injection of piggyBac

A: embryo after $24 \mathrm{~h}$ of injection; B, C: embryo after $48 \mathrm{~h}$ injection;

D, E: 48 hours after the injection gradually developed into 'U' of the embryo

\subsection{Expression of pBac3xP3 EGFP in larval stage}

The green fluorescent embryos of Spodoptera litura were recorded 48 hours after injection, and the embryos without fluorescence were punctured, placed in a constant temperature incubator, and incubated for 96 hours. Under the fluorescence microscope, it was found that not all surviving embryos could be hatched into larvae, and the proportion of larvae that could be hatching larvae was about $113 / 377=29.97 \%$ (see Table 1), and the eggs were hatched Larvae, only a small number of individuals can further observe the green fluorescence, indicating that after microinjection treatment will have a certain negative impact on the embryo. Further observation of the fluorescent larvae revealed that the site of the fluorescence was not expressed in the whole larvae, but there were some specific sites (Figure 3-2), mainly in the head and chest, and also observed at the tail. Indicating that EGFP expression was approximately the same as observed in the larval stage and later in the embryo.

Figure 3-2 Expression of EGFP in Partial Spodoptera litura larvae after injection of piggyBac

\subsection{Expression of $\mathrm{pBac} 3 \times \mathrm{xP} 3 \mathrm{EGFP}$ in adults}

After screening the G0 transgenic positive individuals into moths, the compound eyes of the moth were observed under blue light. The expression of EGFP in the transgenic moth was observed, and the green fluorescence was observed in the compound eyes. The expression of green fluorescence was not observed in the control group. Indicating that the piggyBac transposon has been successfully obtained in this experiment.

Figure 3-3 Expression of EGFP in G0 transgenic moths

A, B: Transgenic Spodoptera litura C, D: control

3.4 Comparison of efficiency of $\mathrm{pBac} 3 \times \mathrm{PP} 3 \mathrm{EGFP}$ and $\mathrm{pBacA} 3$
In order to compare the fluorescence expression rate after injection of two specific promoters, the egg mass was selected within 4 hours postpartum. After two kinds of microinjection, the number of pBac3xp3 EGFP was 1787, and the number of fluorescent eggs was $37721.1 \%$ of the eggs were implanted. Eleven larvae were hatching with $30.0 \%$ of the larvae. Only two larvae expressed EGFP fluorescence, which accounted for $1.7 \%$ of the larvae. Two of the two insects developed to pupae, Moth, indicating that the transposition efficiency is very low.

There were 1605 eggs with pBacA3 EGFP, 637 fluorescent eggs were observed, accounting for $39.7 \%$ of the eggs. The eggs were incubated with 392 larvae, and the hatching rate was 61.5\%. Among them, 10 larvae expressed EGFP fluorescence, 2.6\%; 10 larvae all developed into feather moths (see Table 1). Statistical analysis showed that the fluorescence expression rate of pBacA3 EGFP in injected eggs and the hatching rate of fluorescent eggs were significantly higher than that of $\mathrm{pBac} 3 \mathrm{xP} 3 \mathrm{EGFP}$, which indicated that pBacA3 EGFP was more suitable as transgenic vector of Spodoptera litura.

Table 1 Table of pBac3xP3 EGFP and pBacA3 EGFP after injection

Inflorescence number of eggs

Number of hatching larvae (\%)

Number of fluorescent larvae (\%) Number of fluorescent larvae

$(\%)$

$\begin{array}{llll}\text { pBac3xp3 EGFP } & 1787 & 377 \\ (21.1 \%) \mathrm{a} & 113 & & \\ (30.0 \%) \mathrm{a} & 2 & & \\ (1.7 \%) & & & \\ \text { pBacA3 EGFP } & 1605 & 637 \\ (39.7 \%) \mathrm{b} & 392 & & \\ (61.5 \%) \mathrm{b} & 10 & & \\ (2.6 \%) & & & \end{array}$

Note: the same column marked different significant differences, $\mathrm{P}<0.05$.

3.5 Molecular Detection of 3.5 pBacA3 EGFP Spodoptera litura

Due to the environmental and climatic effects of the breeding process, only 10 hybrids were obtained from the G0 generation. In order to detect whether the piggyBac transposon has been stably inserted into the genome of the genus Spodoptera litura, the hatching of the A3 fluorescent positive insects is kept to 4 or 5 times, and the genomic DNA is extracted and detected by EGFP as the primer. However, there was showing no band at all (as shown in Figure 3-4 lane 3).

And then actin as primer conditions for PCR detection, can be obtained as shown below. Because actin protein is highly conserved in eukaryotes, it is an indispensable component of living tissue and normal life activity. Can be used to detect whether genomic DNA of the positive insects is extracted when the genomic DNA is extracted. The result of PCR analysis showed that there was a specific band (about $510 \mathrm{bp}$ ) in the second lane (ie, A3 fluorescent insects with actin as primers), indicating that there was no problem with the extracted genomic DNA. In the fourth lane, there are also specific bands, and on the 700 bands, it is similar to the size of EGFP (710bp) involved in this laboratory, indicating that there is no problem with the injected plasmid. 
Figure 3-4 PCR identification of transgenic Spodoptera litura

Lane 1: marker Lane 2: A3 Fluorescence actin

Lane 3: A3 Fluorescent Genomic DNA Lane 4: A3 plasmid EGFP

3.6 cDNA analysis of $\mathrm{pBac} 3 \mathrm{xP} 3$ EGFP positive larvae

Since no more $\mathrm{pBac} 3 \mathrm{xP} 3$ EGFP-positive adult individuals were obtained, the relevant genomic DNA was not extracted and identified. And only in the hatchery larvae can be expressed, so the incubation of fluorescent larvae directly RNA extraction, reverse transcription of the cDNA, and then EGFP primers for PCR, the results are as follows: G0 generation of fluorescent cDNA $1 \mathrm{x}$ and G0 generation of fluorescent eggs CDNA 0.1x can produce specific bands, about 700bp on the side, and the laboratory involved in the size of EGFP (710bp) similar to the G0 generation of eggs do have the expression of EGFP, resulting in green fluorescent protein The The third and fifth lanes, A3 genomic DNA $1 \mathrm{x}$ of A3 and genomic DNA diluted to $0.1 \mathrm{x}$ were observed, and no specific bands were produced under the same conditions. Indicating that its gene carrier is not inherited to the next generation, cannot be detected. And the sixth lane to A3 plasmid as a control case, you can clearly see the bright specific band, and is also close to 710bp or so, once again verified the injection of the micro-plasmid is no problem.

Figure 3-5 cDNA detection of pBac3xP3 EGFP-positive individuals

Lane 1: 1200 bp marker, lane 2: G0 generation of fluorescent cDNA $1 \mathrm{x}$,

Lane 3: G0 on behalf of the fluorescent egg cDNA 0.1x, lane 4: A3 of the G1 generation of fluorescent insect genomic DNA 1x,

Lane 5: A3 of the G1 generation of the genomic DNA $0.1 \mathrm{x}$, Lane 6: A3 plasmid.

On the other hand, due to the molecular biology of insects, some markers are often used to detect the quality of tissue cDNA templates. One of the most commonly used marker gene is actin 3 (actin3), belonging to the ARP family actin subfamily [8]. Actin protein is highly conserved in eukaryotes and is an indispensable component of living tissue and normal life activities [9]. Therefore, in the EGFP as the purpose of the band at the same time, we also carried out to actin 3 for the purpose of the PCR detection, the results of electrophoresis results shown in Figure 3-6. It can be seen from the figure that the fluorescent protein and the fluorescent larvae of the G0 generation can be reproduced in the above PCR system, and the obvious specific bands (lanes 2 to 5) can be amplified and the bands The size of between $500-700 \mathrm{bp}$, close to $500 \mathrm{bp}$, and Actin3 size consistent, indicating that different times in the positive insects can be expressed, and little change.

Figure 3-6 cDNA detection of pBac3xP3 EGFP positive individuals

Lane 1: 1200bp marker Lane 2: G0 Generation Embryos cDNA Lane 3: 0.1xG0 Generation Embryos cDNA

Lane 4: G0 larvae cDNA lane 5: 0.1xG0 generation larvae cDNA

Note: Actin as primer

\section{Discussion}

4.1 EGFP can be expressed normally in Spodoptera litura In this study, the $3 \mathrm{xP} 3$ promoter and the actin $\mathrm{A} 3$ promoter, the enhanced green fluorescent protein gene and the SV40 polyadenylation recognition sequence were used as elements and inserted into the piggyBac transposable vector. Spodoptera littoralis pBacA3 EGFP and pBac3xP3 EGFP transgenic vectors. Since the promoter of the silkworm actin A3 is a strong promoter, it is possible to efficiently transcribe downstream genes, and by injecting it into the moth eggs in the early stage of the blastoderm formation, it is strongly detected on the second day of embryonic development Of the green fluorescence, indicating that the constructed vector can correctly express EGFP gene [10].

4.2 Comparison of two different promoters

In this study, the transgenic vector pBac3xp3 EGFP and the helper plasmid $\mathrm{p} 3 \mathrm{xP} 3 \mathrm{H}$ were introduced into the early embryos of Spodoptera litura, and the positive larvae of Transgenic Spodoptera litura were obtained on G0 generation. A microinjection control of pBacA3 EGFP was also performed.

In this experiment, Spodoptera litura A3 was used as promoter and $3 \times p 3$ promoter, EGFP was the foreign gene. The expression of downstream genes was observed by fluorescence observation of different developmental stages of Transgenic Spodoptera litura. The results showed that the pBacA3 EGFP gene was expressed in all developmental stages of Spodoptera litura, which was consistent with the expression of A3 actin expressed as A3 as a broad-spectrum promoter in all tissues and stages of development. EGFP-specific fragments were not amplified in the progeny of fluorescent expression. The integration of exogenous genes with host DNA is an extremely complex process, especially for eukaryotic transgenes, which has not yet been elucidated. In general, foreign genes into the eukaryotic cell receptor after a variety of whereabouts: then degradation of loss, retention in the cytoplasm or effectively into the nucleus. Intentional nuclei are also likely to be degraded, and are free to exist within the nucleus and integrate into the host chromosome (both randomized and effectively integrated at a specific location). If there is a certain rule that exogenous DNA is absorbed and incorporated into its own system, the effective integration frequency should be specific regardless of the method. Otherwise the integration may be a completely randomized biological process that can be detected by PCR amplification, Southem hybridization [11]. The reason why this experiment failed to amplify the EGFP-specific fragment was probably due to the fact that the G0 generation was a chimera, and the transferred pBacA3 EGFP was too small for the genome of the whole Spodoptera litura and the concentration of the EGG too low, resulting in a very good amplification of the results. The specific research needs further verification. Similarly, pBac3xP3 EGFP positive individuals is too small, cannot play a better description of the effect, but in the positive larvae can be expressed in the eye, and $3 \times \mathrm{XP} 3$ start the action is consistent.

4.3 Difficulty in obtaining positive individuals

The first key to successful access to transgenic Spodoptera litura was to import exogenous DNA correctly into the early embryos of Spodoptera litura and as close as possible to the fertilized nucleus in the middle of the embryo. This is because the microinjection will cause greater mechanical damage to the moth eggs, and the introduction of foreign liquids will affect the normal development of the embryo, making the larvae after injection of egg survival rate is low. In the transgenic injection experiments found that the moth eggs after the embryo in the late development of the embryo, hatching cannot be too much because of the eggs cannot bite the suffocation. Second, the larvae after hatching larvae are also significantly weaker 
than normal, with low resistance, susceptible to disease, and are not prone to oviposition mating stage. Although the first use of piggyBac vector to obtain transgenic insects has been reported for almost four years, but the world is still only a few laboratories can successfully carry out transgenic insect operations. The main reason is that the survival rate of the transgenic moth injection and normal growth and development rate is low. Therefore, the study of genetically modified Spodoptera litura by microinjection requires more skillful microinjection technique, which minimizes the loss of moth eggs content during injection, which is the key to improve the hatching rate and survival rate of injection moth eggs. In this experiment, the expression of fluorescence was observed in about $60 \%$ of the moth eggs injected in some experiments, indicating that the introduction of exogenous DNA and the early development of embryos were normal. Since EGFP has a peak at $488 \mathrm{~nm}$, it can be excited by more harmless blue light, and its fluorescence intensity is about 35 times higher than that of wild type GFP, suitable for rapid and no damage detection. The experiment also found that by transient ultraviolet radiation, and did not affect the subsequent development of embryos. The selection of these individuals that can detect the fluorescence as a candidate transgenic positive individual will also greatly improve the final transgenic success rate and shorten the transgenic cycle. The rapid expression system of exogenous EGFP gene reported in this paper is not only the first step necessary to successfully carry out the transgenic gene of Spodoptera litura, but also itself can be applied to the study of gene function, such as replacing the A3 promoter with the gene regulation Sequence, which can be very convenient in time and space to locate the expression of the gene, and to be studied gene coding sequence to replace the EGFP coding region or inserted into its upstream or downstream, but also to study the gene on the embryonic development process [12].

4.4 Selection of transposon systems

As the most widely available piggyBac transposon in the insect transgene now, it has been shown that more than 20 insects can be used in 5 heads (Lepidoptera, Diptera, Hymenoptera, Coleoptera and Orthoptera), Which studies more adequate transgenic techniques for silkworms. Comparative genomic analysis showed that piggyBac-like elements were present in genomic sequences of a variety of organisms, suggesting that piggyBac transposon elements have transposon activity in a variety of organisms. In recent years, it has been found that endogenous piggyBac transposons have been successfully detected in a variety of insects such as Spodoptera litura, Lepidoptera, Cynomolgus, and so on. The results of sequence alignment showed that the similarity of piggyBac and IFP2 in Prodenia litura was 91\%, which belonged to IFP2 highly similar sequence [13]. These suggest that piggyBac may have transposon activity in the body of the genus Spodoptera. The results of this experiment well proved this, but the efficiency of the different promoters is different. The plasmid for pBacA3 EGFP in this laboratory was relatively mature for the plasmids of the two promoters in the experiment.

4.5 Comparison of Transgenic Spodoptera litura and Silkworm

The first access to genetically modified silkworms, using pBacA3 EGFP, also confirmed that pBacA3 EGFP was useful in the screening of the G1 generation larvae, but had to feed a large number of silkworms. The biggest drawback of this promoter was time consuming. EGFP expression was observed in the G0 chimeric larvae under the Bm-Actin3 EGFP labeling, and was also observed in the offspring of transgenic larvae and G0-depleted yellowing cells, but not in G0 embryos or offspring. Thus, under the A3 promoter, it is not possible to screen for transgenic individuals prior to G1 larvae. However, EGFP can be observed in the eggs of the G0 generation of Spodoptera litura, which can be used as screening vouchers [14].

In addition, since the 3xP3-EGFP marker has been shown to be a universal marker for a suitable transgenic insect, it is very effective in converting silkworm germ cells into embryonic stage screening using piggyBac transgenic vector $\mathrm{pBac} 3 \mathrm{xP} 3$ EGFP from embryonic development The five-day central and peripheral nervous system produces EGFP green fluorescent protein, which is expressed at a high level throughout the larvae stage, as well as in the compound tissues of the compound eyes and pupae. In this experiment, pBac3xP3 EGFP was used, and it was possible to roughly judge the expression in some tissues during the screening process. Genomic DNA extraction could also be carried out by tissue, which could improve the extraction efficiency. However, the process of obtaining positive individuals was difficult. Therefore, the preliminary determination in the genus Spodoptera transgenic technology, the use of pBacA3 EGFP transposon system is a better choice.

4.6 Expression of exogenous genes

The expression level of exogenous genes is critical to its role in transgenic gene. There are many factors that may affect the expression level, such as the promoter, the coding sequence, the copy number, and the insertion position. Whether the expression of the exogenous gene in the host depends on: (1) whether it is located in the expressible system in the downstream of the promoter; (2) whether it is in the active state in time, and to ensure that the foreign gene is fully expressed Potential [16].

In the current insect transgenic study, most of the enhanced green fluorescent protein EGFP as a positive individual screening markers. Since the expression of the fluorescent protein is affected by the promoter's ability to start, the position and copy number of the inserted fragment in the host genome, the codon bias of the host, and so on, thus affecting the screening efficiency of the transgenic silkworm. From the results of this study, it is desirable to select the actin A3 promoter to activate EGFP markers with longer excitation wavelengths. However, most studies have shown that DsRed has a screening marker, and good results can be obtained [17-18]. Therefore, the next step of the study can explore the EGFP and DsRed two markers in the Spodoptera litura in the screening effect. But it is difficult to avoid the screening process of ultraviolet radiation on the embryo, so if you can choose early in the embryo can be observed by the phenotypic mutant gene to replace the fluorescent marker gene, is expected to get better screening results and efficiency.

4.7 Genetic Chimerism of Transgenic Genus Spodoptera litura

Spodoptera littoralis fertilized eggs began to spawning about $3 \mathrm{~h}$ after spawning, microinjection if it is completed in the fertilized egg before cleavage, the injection of foreign genes into the nuclear genome without duplication of the site, with the host gene Replication, with the host nucleus splitting into the nucleus [19-20]. Since the nuclei have not yet been split, each nucleus of the fertilized egg cleavage can obtain the same copy of the foreign gene. The resulting transgenic Spodoptera litura should be homozygous. In general, the probability of producing the first homozygote for mammalian progeny is larger; it is also theoretically feasible for transgenic insects, as long as the plasmid DNA is injected into the egg before cleavage and the transposon carries. The source gene can be homozygous when it is integrated into its genome before fertilized egg cleavage. However, in practice, it is very difficult to obtain the first homozygous in transgenic insects. First, due to the presence of insect eggshell and cytoplasm opaque, cannot 
see the internal nucleus, it is difficult to accurately inject exogenous DNA into the nucleus, and genetically modified insects are basically cytoplasmic injection. Injection, only according to the general judgment, the transposon plasmid injected in the vicinity of the nucleus, the use of transposon transposition and insertion function, carrying foreign gene integration into the genome, when the male and female protoplasts formed zygote, fertilized eggs into mitosis, it is difficult to assure integration at the fixed site of each cell at this time. Second, insect egg cleavage fast, and insects spawning a long time, it is difficult to accurately determine when the eggs really fertilization. Insect eggs early division of the mitotic non-split, injection is difficult to ensure that just in the prokaryotic period, may have occurred several times split, this time cannot get homozygous offspring.

In this study, the positive transgenic individuals were obtained 3 $\mathrm{h}$ after spawning and after $3 \mathrm{~h}$ after the injection operation obtained. Thus, positive transgenic individuals are more likely to be chimeric. Homozygous transgenic individuals are not critical in the transgenic system. Whether the gene can be integrated into germ cells is the key to achieving stable inheritance of transgenic Spodoptera litura. In addition to somatic cell chimerism, germ cells also have chimeric phenomena, which makes the first generation can detect EGFP transgenic Spodoptera litura, the transgene may also not inherited or only very low frequency of genetic. This requires the establishment and maintenance of a good transgenic lineage program.

Other issues affecting the establishment of transgenic lines cannot be ignored, genetically modified insects may exist with the integration of genetically modified or functional, poor fertility, phenotype is not ideal and other issues, therefore, to compensate for the high proportion of insect chimerism and other issues, a large number of eggs, in order to obtain a large number of first generation of transgenic insects and their offspring for screening.

\section{REFERENCES}

[1] Isobe R, KojimaK, Matsuyama T, et al. Use of RNAi technology to confer enhanced resistance to BmNPV on transgenic silkworms [J]. Arch Virol. 2004, 149 (10): 1931-1940.

[2] Imamura M, Nakai J, Inoue S, et al. Targeted gene expression using the GAL4 / UAS system in the silkworm Bombyx mori [J]. Genetice. 2003, 165 (3): 1329-1340.

[3] Liu Chun, Xu Hanfu, Chen Yulin, Li Chunfeng, Zhang Meirong, Xia Qingyou. Transgenic research of silkworm and the expression of actin A3 promoter [J]. Journal of Silkworm Research, 2007,27 (3): 6.

[4] Tamura T, Thibert C, Roya C, Kanda T, Abraham E, Kamba M, K?motoN. Et al. Germline transformation of the silkworm Bombyx mori L. using a piggyBac transposon-derived vector [J]. Nat Biotechnol, 2000, 18: 81-84.

[5] Tomita M, Munetsuna H, Sato T, Adachi T, Hino R, Hayashi M, ShimizuK et al. Transgenic silkworms produce recombinant human type III procollagen in cocoons [J]. Nat Biotechnol 2003, 21: 52-56.

[6] Qin Houguo, Ye Zhengxiang. Spodoptera litura catastrophe law and control [M]. Beijing: China Agricultural Science and Technology Press, 2007 edition.

[7] Siddharth Tiwari, Devesh K. Mishra, Ankit Singh, PK Singh, Rakesh Tuli. Expression of a synthetic cry1EC gene for resistance against Spodoptera litura in transgenic peanut (Arachis hypogaea L.) [M]. Plant Cell Rep, 27: 1017-1025.

[8] Holly V. Goodson and William F. Hawse. Molecular evolution of the actin family [J]. Cell Science at a Glance: 2619-2622.

[9] Chai Chunli, Li Yuxin, Meng Meng, Zuo Weidong. Identification of nonspecific amplified fragments of Actin3 primer in silkworm gland and optimization of A3 primer amplification conditions [J] .Journal of Silkworm, 2009,29 (4) 7-12.

[10] $\mathrm{Xu} \mathrm{H}$, et al. Construction and expression of transgenic vector pBacA3EG in silkworm [J]. ACTA ENTOMOLOGICA SINICA, 2005,48 (5): 799 - 803 (in Chinese with English abstract)

[11] Rong Rui, Li Zhenang. Advances in transgenic research of silkworm [J]. Insect knowledge [M], 1998,35 (3): 189-191.

[12] Xu Hanfu, Xia Qingyou, Liu Chun, Wu Xuefeng, Yang Yuanping, Zhao Ping, Xiang Zhonghuai. Construction and expression of transgenic vector pBacA3EG in silkworm [J]. ACTA ENTOMOLOGICA SINICA, 2005,48 (5):
$799-803$.

[13] Wu Min. Study on piggyBac transposon in Lepidoptera insects [M]. Nanjing: Nanjing Agricultural University, 2008.

[14] Thomas JL, Da Rocha M, Besse A, Mauchamp B, Chavancy G. 3xP3EGFP marker facilitates screening for transgenic silkworm Bombyx mori L. from the embryonic stage onwards [J] Insect Biochem Mol Biol. 2002 Mar 1; 32 (3): 247-53.

[15] Tamura T, Thibert C, Roya C, Kanda T, Abraham E, Kamba M, K?motoN. Et al. Germline transformation of the silkworm Bombyx mori L. using a piggyBac transposon-derived vector [J]. Nat Biotechnol, 2000 , 18: 81-84.

[16] Xu Hanfu, Li Juan, Liu Chun, Xia Qingyou. Advances, application and prospect of insect transgenic research [J]. Journal of Silkworm Research, 2004,24 (4): 19-26.

[J]. ACTA ENTOMOLOGICA SINICA, 2009,52 (6): 595-603. [J]. ACTA ENTOMOLOGICA SINICA, 2009,52 (6): 595-603. [J]. ACTA ENTOMOLOGICA SINICA, 2009,52 (6): 595-603. Effects of Several Factors on Transgenic Efficiency in Silkworm [J].

[18] Carsten Horn, Bernhard G. M. Schmid, Frank S. Pogoda, Ernst A. Wimmer. Fluorescent transformation markers for insect transgenesis [J]. Insect Biochemistry and Molecular Biology, 2002, 32: 1221-1235.

[19] Zhang Qunling, Jia Fenglong, Li Cuiying, Lu Aiping. Embryonic development of Spodoptera litura. East China Insects, 1998,7 (1): 51-54.

[20] ZHANG Qun-ling, JIA Feng-long, LI Cui-ying, LU Ai-ping. Study on ultrastructure of embryonic development of egg shell and body wall of Spodoptera litura Fabricius [J]. East China Entomology, 1999,8 (2): 4-5.

[21] Tomita M, Hino R, Ogawa S, Iizuka M, Adachi T, Shimizu K, Sotoshiro H, Yoshizato K. A germline transgenic silkworm that secretes recombinant proteins in the sericin layer of cocoon[J].Transgenic Res,2007,16:449-465. 